\title{
Equivalence operators that are associative
}

\author{
József Dombi \\ University of Szeged \\ Department of Informatics \\ e-mail: dombi@inf.u-szeged.hu
}

\begin{abstract}
We begin with a paradox of the equivalence relation, and we solve it by using the neutral value of the negation. The so-called Pliant equivalence operator fulfils the modified requirements of the fuzzy equivalence relations. After, we study two models of the equivalence operator. We show that in the Pliant operator case the natural extension of two expressions is equivalent. It has two different types of transitivity. It is associative, and it can be extended to many variables. On this basis, we can create the weighted form of the equivalence operator.
\end{abstract}

Keywords: equivalence operator, symmetric difference operator, similarity relation, indistinguishability, T-equivalence

\section{Introduction}

Fuzzy equivalence relations were introduced by Zadeh [7] as a generalization of the concept of an equivalence relation. Since then, they have been widely studied (e. g. to measure the degree of indistinguishability or similarity between the objects of a given universe of discourse) and they have been shown to be useful in different contexts such as fuzzy control, approximate reasoning and fuzzy cluster analysis. Depending on the authors and the context in which they appeared, they had alternative labels such as similarity relations [36], indistinguishability operators $[7,14,15,24,25,26,33]$, T-equivalences $[2,3]$ and many-valued equivalence relations $[12,13]$. In [11], the authors investigate various properties of equivalence classes of fuzzy equivalence relations over a complete residuated lattice.

Bodenhofer [6] in his article presented an alternative concept of fuzzy orderings. With his viewpoint of representation and construction, he adopted a generalized approach. He stated an important relationship between fuzzy ordering and approximate similarity and also found a fundamental connection between orderings and equivalence relations. In his article, he uses the classical definitions to establish the new results.

Definition 1. An associative, commutative, and non-decreasing binary operation on the unit interval (i.e. $a[0 ; 1]^{2} \rightarrow[0 ; 1]$ mapping) which has 1 as neutral 
element is called triangular norm, for short, t-norm. A t-norm $T$ is called left-continuous if and only if all partial mappings $T(x,$.$) and T(., x)$ are leftcontinuous.

The continuous, strictly monotonously increasing Archimedean t-norm $(t(x, y))$ and t-conorm $(s(x, y))$ can be represented by additive generator functions that have the following form

$$
t(x, y)=f_{c}^{-1}\left(f_{c}(x)+f_{c}(y)\right)
$$

and

$$
s(x, y)=f_{d}^{-1}\left(f_{d}(x)+f_{d}(y)\right) .
$$

The generator functions are determined up to a multiplicative factor [28].

Definition 2. For a left-continuous t-norm $T$, the residual implication (residuum) $\vec{T}$ is defined as

$$
\vec{T}(x, y)=\sup \{u \in[0,1] \mid T(u, x) \leq y\}
$$

Lemma 1. (Fodor and Roubens [19], Gottwald [22], Hájek [23]). Consider a left-continuous t-norm $T$. Then the following holds for all $x, y, z \in[0,1]$ :

1. $x \leq y \quad$ if and only if $\vec{T}(x, y)=1$;

2. $T(x, y) \leq z \quad$ if and only if $x \leq \vec{T}(y, z)$;

3. $T(\vec{T}(x, y), \vec{T}(y ; z)) \leq \vec{T}(x, z)$;

4. $\vec{T}(1, y)=y$,

5. $T(x, \vec{T}(x, y)) \leq y$,

6. $y \leq \vec{T}(x, T(x, y))$ :

Furthermore, $\vec{T}$ is non-increasing and left-continuous in the first argument and non-decreasing and right-continuous in the second argument.

Analogously to the Boolean case, we can also use a residual implication to define a concept of logical equivalence.

Definition 3. The bi-implication $\stackrel{\leftrightarrow}{T}$ of a left-continuous t-norm $T$ is defined as

$$
\stackrel{\leftrightarrow}{T}(x, y)=T(\vec{T}(x, y), \quad \vec{T}(y, x)):
$$

Lemma 2. For a left-continuous t-norm $T$, the following assertions hold (for all $x, y, z \in[0 ; 1])$ :

1. $\stackrel{\leftrightarrow}{T}(x, y)=1$ if and only if $x=y$;

2. $\stackrel{\leftrightarrow}{T}(x, y)=\min (\vec{T}(x, y), \vec{T}(y, x))$;

3. $\stackrel{\leftrightarrow}{T}(x, y)=\overleftrightarrow{T}(y, x)$ 
4. $T(\overleftrightarrow{T}(x, y), \overleftrightarrow{T}(x, y)) \leq \overleftrightarrow{T}(x, y)$

5. $\stackrel{\leftrightarrow}{T}(x, y)=\vec{T}(\max (x, y), \min (x, y))$

In classical logic the simplest operation is the equivalence relation, but there are problems introducing it in the continuous-valued case. An equivalence relation has to meet the following requirements:

In our article the negation operator plays an important role, and we denoted it by $\eta(x)$. Let us denote the fix point of the negation by $\nu_{*}$ (i. e. $\eta_{\nu_{*}}\left(\nu_{*}\right)=\nu_{*}$ ). The developed equivalence operator depends on $\nu_{*}$ and we denote it by $e_{\nu_{*}}(x, y)$

1. $e_{\nu_{*}}:[0,1] \times[0,1] \rightarrow[0,1]$ is a continuous mapping

2. $e_{\nu_{*}}(0,0)=1 \quad e_{\nu_{*}}(1,1)=1$

3. $e_{\nu_{*}}(0,1)=0 \quad e_{\nu_{*}}(1,0)=0$,

i.e. it must be compatible with two-valued logic. 2 and 3 can be generalized to:

4. $e_{\nu_{*}}(x, x)=1$

5. $e_{\nu_{*}}\left(x, \eta_{\nu_{*}}(x)\right)=0$

On the one hand, 4 is very natural and all theoreticians agree on it. On the other, they forget that 5 should be as important as 4 . Demanding that 4 and 5 be simultaneously satisfied leads to a paradox.

Lemma 3 (The paradox of the equivalence relation). There is no equivalence relation which fulfils 4 and 5.

Proof. Let $\nu_{*}$ be the fix point of $\eta_{\nu_{*}}(x)$. Then from $4, e_{\nu_{*}}\left(\nu_{*}, \nu_{*}\right)=1$ and from $5 e_{\nu_{*}}\left(\nu_{*}, \eta_{\nu_{*}}\left(\nu_{*}\right)\right)=e_{\nu_{*}}\left(\nu_{*}, \nu_{*}\right)=0$.

In this article, we solve this paradox and we use $\nu_{*}$ ad a threshold by modifying the requirements 1-3. All the results are derived using the Pliant system.

The question here is, which value is reasonable for $e_{\nu_{*}}\left(\nu_{*}, \nu_{*}\right)$ ? If we recall that the values represent uncertainties and we hesitate whether object $\mathrm{A}$ has the property $x_{A}\left(=\nu_{*}\right)$ and object $\mathrm{B}$ has the property $x_{B}\left(=\nu_{*}\right)$, then we are also not sure that $\mathrm{A}$ and $\mathrm{B}$ are equivalent from this point of view. Our suggestion is

$$
e_{\nu_{*}}\left(\nu_{*}, \nu_{*}\right)=\nu_{*} .
$$

i. e. in very noisy cases we cannot say whether these two are equivalent. In the next section we give a brief description of the Pliant concept. 


\section{Pliant systems}

In Pliant systems, we focus on the DeMorgan systems which have infinitely many negation operators. These types of operators are important because the fix point of the negation (see Eq.(1) later) can be varied, this value can be interpreted as a decision level and this kind of logic is very flexible. Such logic is important in different areas. Cintula et al. [10] focus on fuzzy logic with an additional involutive negation operator, but in our case we have infinitely many negation operators that satisfy the DeMorgan identity [16, 18]. In my articles, I showed that this operator class has important properties.

From an application's point of view, strictly monotonously increasing operators are useful and they have many applications. This is why we will focus on strictly monotonously increasing operators of the Pliant concept.

\section{Triangular norms and conorms}

Definition 4. We say that $\eta(x)$ is a negation operator if $\eta:[0,1] \rightarrow[0,1]$ satisfies the following conditions:

$$
\begin{aligned}
& C 1: \eta:[0,1] \rightarrow[0,1] \text { is continuous; } \\
& C 2: \eta(0)=1, \eta(1)=0 \\
& C 3: \eta(x)<\eta(y) \text { for } x>y \\
& C 4: \eta(\eta(x))=x
\end{aligned}
$$

From $\mathrm{C} 1$ and $\mathrm{C} 3$ it follows that there exists a fix point $\nu_{*} \in[0,1]$ of the negation, where

$$
\eta\left(\nu_{*}\right)=\nu_{*} .
$$

In the following, we construct a subset of t-norms and t-conorms which fulfil some special conditions and we will call them pliant operators. These two classes of operators will be denoted by $c(x, y)$ (fuzzy AND operator) and $d(x, y)$ (fuzzy OR operator), not $t(x, y)$ and $s(x, y)$.

In the Pliant concept, we will characterize the operator class (strict t-norm and strict t-conorm) for which various negation operators exist and build a DeMorgan class, i. e. $\eta(c(x, y))=d(\eta(x), \eta(y))$.

The fix point $\nu_{*}$ or the neutral value $\nu_{*}$ can be regarded as a decision threshold. Operators with various negation operators are useful because the threshold value can be varied.

The operators $c(x, y)$ and $d(x, y)$ build a DeMorgan system for $\eta_{\nu_{*}}(x)$, where $\eta_{\nu_{*}}\left(\nu_{*}\right)=\nu_{*}$ for all $\nu_{*} \epsilon(0,1)$ if and only if there exists an additive generator $f_{c}(x)$ and $f_{d}(x)$ of $c(x, y)$ and $d(x, y)$, respectively

$$
f_{c}(x) f_{d}(x)=1,
$$


where $f_{c}(x)$ and $f_{d}(x)$ are the generator functions of the strict t-norms and t-conorms. A proof of this can be found in [18].

Knowing $f_{c}(x)$ or $f_{d}(x)$ means that we automatically know the other, since they are the reciprocals of each other.

Definition 5. Such system where Eq. (2) holds is called a Pliant system.

Hence the general form of a pliant system is

$$
\begin{aligned}
o_{\alpha}(x, y) & =f^{-1}\left(\left(f^{\alpha}(x)+f^{\alpha}(y)\right)^{1 / \alpha}\right) \\
\eta_{\nu_{*}}(x) & =f^{-1}\left(\frac{f^{2}\left(\nu_{*}\right)}{f(x)}\right)
\end{aligned}
$$

where $f(x)$ is the generator function of the strict t-norm and $f:[0,1] \rightarrow[0, \infty]$ is a continuous and strictly decreasing function. Depending on the sign of $\alpha$, the operator is conjunctive or disjunctive.

$$
\text { If } \alpha>0, \quad \text { then }
$$

1.

$$
\begin{aligned}
& o_{\alpha}(x, y) \quad \text { is a conjunctive operator (t-norm) } \\
& \text { If } \alpha<0, \quad \text { then }
\end{aligned}
$$

2 .

$$
o_{\alpha}(x, y) \quad \text { is a disjunctive operator (t-conorm) }
$$

Let $c(x, y)=f^{-1}(f(x)+f(y))$, then

$$
d(x, y)=f^{-1}\left(\frac{1}{\frac{1}{f(x)}+\frac{1}{f(y)}}\right)
$$

We can summarize the elements of the Pliant system (operators and their correspondent weighted form) like so:

$$
\begin{gathered}
c(\mathbf{x})=f^{-1}\left(\sum_{i=1}^{n} f\left(x_{i}\right)\right) \quad c(\mathbf{w}, \mathbf{x})=f^{-1}\left(\sum_{i=1}^{n} w_{i} f\left(x_{i}\right)\right) \\
d(\mathbf{x})=f^{-1}\left(\frac{1}{\sum_{i=1}^{n} \frac{1}{f\left(x_{i}\right)}}\right) d(\mathbf{w}, \mathbf{x})=f^{-1}\left(\frac{1}{\sum_{i=1}^{n} \frac{w_{i}}{f\left(x_{i}\right)}}\right) \\
\eta(x)=f^{-1}\left(\frac{f^{2}\left(\nu_{*}\right)}{f(x)}\right),
\end{gathered}
$$

where $f(x)$ is the generator function of the strict t-norm.

The Pliant system has been recently extended. In this system, the operator class of the uninorms is called the aggregative operator [16]. A special case of 
the Pliant system is when the generator function is given by the Dombi operator.

\section{The operator system of Dombi}

The Dombi operators [16] form a pliant system and the operators are:

$$
\begin{gathered}
c_{\alpha}(\mathbf{x})=\frac{1}{1+\left(\sum_{i=1}^{n}\left(\frac{1-x_{i}}{x_{i}}\right)^{\alpha}\right)^{1 / \alpha}} c_{\alpha}(\mathbf{w}, \mathbf{x})=\frac{1}{1+\left(\sum_{i=1}^{n} w_{i}\left(\frac{1-x_{i}}{x_{i}}\right)^{\alpha}\right)^{1 / \alpha}} \\
d_{\alpha}(\mathbf{x})=\frac{1}{1+\left(\sum_{i=1}^{n}\left(\frac{1-x_{i}}{x_{i}}\right)^{-\alpha}\right)^{-1 / \alpha}} \quad d_{\alpha}(\mathbf{w}, \mathbf{x})=\frac{1}{1+\left(\sum_{i=1}^{n} w_{i}\left(\frac{1-x_{i}}{x_{i}}\right)^{-\alpha}\right)^{-1 / \alpha}} \\
\bar{c}_{\alpha}(\mathbf{x})=\frac{1}{1+\left(\frac{1}{n} \sum_{i=1}^{n}\left(\frac{1-x_{i}}{x_{i}}\right)^{\alpha}\right)^{1 / \alpha}} \quad \bar{d}_{\alpha}(\mathbf{x})=\frac{1}{1+\left(\frac{1}{n} \sum_{i=1}^{n}\left(\frac{1-x_{i}}{x_{i}}\right)^{-\alpha}\right)^{-1 / \alpha}} \\
\eta_{\nu_{*}}(x)=\frac{1}{1+\left(\frac{1-\nu_{*}}{\nu_{*}}\right)^{2} \frac{x}{1-x}},
\end{gathered}
$$

where $\left.\nu_{*} \in\right] 0,1[$, with generator functions

$$
f_{c}(x)=\left(\frac{1-x}{x}\right)^{\alpha} \quad f_{d}(x)=\left(\frac{1-x}{x}\right)^{-\alpha},
$$

where $\alpha>0$. The operators $c, d$ and $\eta$ fulfil the De Morgan identity for all $\nu$. In the next part, we will use the notation $\bar{c}_{1}(\mathbf{x})=\bar{c}(\mathbf{x})$ and $\bar{d}_{1}(\mathbf{x})=\bar{d}(\mathbf{x})$

\section{Symmetric difference and equivalence operators}

In Boolean algebra, we may use several equivalent expressions to represent the symmetric difference

$$
\begin{aligned}
& x \triangle_{1} y=(x \wedge \bar{y}) \wedge(y \wedge \bar{x}) \\
& x \triangle_{2} y=(x \vee y) \wedge \overline{(y \wedge x)}
\end{aligned}
$$

From the very beginning [8], the symmetric difference, i. e. the representation of the exclusive or, has been an important operation. However, to date there have been relatively few articles on it. One notable article is by Alsina and Trillas [1]. 
In fuzzy logic, one can model the symmetric difference based on conjunction, disjunction (t-norm, t-conorm) and negation operators in the unit interval $[0,1]$. That is,

$$
\begin{aligned}
& s^{(1)}(x, y)=d(c(x, \eta(x)), c(y, \eta(x))) \quad \text { or } \\
& s^{(2)}(x, y)=c(d(x, y), \eta(c(x, y))),
\end{aligned}
$$

where $d$ is the disjunctive operator (t-conorm), $c$ is the conjunctive operator (t-norm) and $\eta$ is a strong negation. The symmetric difference operator is important because using this operator we can introduce metrics, and with these metrics we can measure the similarity. From an application point of view, it is very useful in problems involving matching and image retrieval $[5,27,34,35,37]$

Using the Pliant operators, we proved the following proposition.

Proposition 1. The weighted Pliant difference operator is

$$
s_{\nu_{*}}(u, x ; v, y)=f^{-1}\left(\frac{1}{2} \frac{f^{2}\left(\nu_{*}\right)+f(x) f(y)}{u f(y)+v f(x)}\right),
$$

where $f(x)$ is the generator function of the strict $t$-norm and $f:[0,1] \rightarrow[0, \infty]$ is a continuous and strictly decreasing function.

Proof. Proof can be found in [17].

\section{Special cases}

If $u=0$ and $v=1$, then

$$
s_{s_{\nu_{*}}}(0, x ; 1, y)=f^{-1}\left(\frac{1}{2}\left(\frac{f^{2}\left(\nu_{*}\right)}{f(x)}+f(y)\right)\right)=\bar{c}(\eta(x), y) .
$$

If $u=1$ and $v=0$, then

$$
s_{s_{\nu_{*}}}(1, x ; 0, y)=f^{-1}\left(\frac{1}{2}\left(\frac{f^{2}\left(\nu_{*}\right)}{f(y)}+f(x)\right)\right)=\bar{c}(x, \eta(y))
$$

If $u=\frac{1}{2} \quad$ and $\quad v=\frac{1}{2}$, then

$$
s\left(\frac{1}{2}, x ; \frac{1}{2}, y\right)=f^{-1}\left(\frac{f^{2}\left(\nu_{*}\right)+f(x) f(y)}{f(x)+f(y)}\right) .
$$

The most common way of introducing the equivalence relation is by using the implication operator. In classical logic, the equivalence is defined by

$$
x \equiv y \quad \text { if and only if }(x \rightarrow y) \wedge(y \rightarrow x),
$$

which can be translated by using

$$
x \rightarrow y=\bar{x} \vee y
$$

Then we get

$$
\begin{aligned}
& x \equiv_{1} \quad y \quad=\quad(\bar{x} \vee y) \wedge(\bar{y} \vee x) \quad \text { or } \\
& x \quad \equiv_{2} y=(x \wedge y) \vee(\bar{x} \wedge \bar{y}) .
\end{aligned}
$$


In the classical two-valued logic, (23) and (24) are equivalent.

Because (23) and (24) are simply the negation of (13) and (14), respectively, we can define them by their negated form. That is,

$$
\begin{aligned}
& \overline{(x \wedge \bar{y}) \vee(\bar{x} \vee y)}=(\bar{x} \vee y) \wedge(x \vee \bar{y}) \\
& \overline{(x \vee y) \wedge(\overline{x \wedge y})}=(x \wedge y) \vee(\bar{x} \vee \bar{y})
\end{aligned}
$$

Given the symmetric difference operator, it is not hard to construct an equivalence operator using the negation operator.

In our notation,

$$
\begin{aligned}
& e_{\nu_{*}}^{(1)}(x, y)=\eta_{\nu_{*}}\left(s_{\nu_{*}}^{(1)}(x, y)\right), \\
& e_{\nu_{*}}^{(2)}(x, y)=\eta_{\nu_{*}}\left(s_{\nu_{*}}^{(2)}(x, y)\right) .
\end{aligned}
$$

Proposition 2. The weighted Pliant equivalence operator is

$$
e_{\nu_{*}}(u, x ; v, y)=f^{-1}\left(2 f^{2}\left(\nu_{*}\right) \frac{v f(x)+u f(y)}{f^{2}\left(\nu_{*}\right)+f(x) f(y)}\right)
$$

when $u=1$ and $v=0$

$$
e_{\nu_{*}}(0, x ; 1, y)=f^{-1}\left(2 f^{2}\left(\nu_{*}\right) \frac{f(y)}{f^{2}\left(\nu_{*}\right)+f(x) f(y)}\right)
$$

when $u=0$ and $v=1$

$$
e_{\nu_{*}}(1, x ; 0, y)=f^{-1}\left(2 f^{2}\left(\nu_{*}\right) \frac{f(x)}{f^{2}\left(\nu_{*}\right)+f(x) f(y)}\right) .
$$

when $\quad u=\frac{1}{2} \quad$ and $\quad v=\frac{1}{2}$,

$$
e_{\nu_{*}}\left(\frac{1}{2}, x ; \frac{1}{2}, y\right)=f^{-1}\left(\frac{f(x)+f(y)}{f^{2}\left(\nu_{*}\right)+f(x) f(y)}\right),
$$

where $f(x)$ is the generator function of the strict $t$-norm and $f:[0,1] \rightarrow[0, \infty]$ is a continuous and strictly decreasing function.

When $u=\frac{1}{2}$ and $v=\frac{1}{2}$, then we will denote $e_{\nu_{*}}\left(\frac{1}{2}, x ; \frac{1}{2}, y\right)$ by $e_{\nu_{*}}(x, y)$.

Proof. The proof is based on Eqs. (7), (17), (25) and (26).

Proposition 3. The mean Pliant equivalence operator is the same as the Pliant equivalence operator. That is,

$$
e_{\nu_{*}}(x, y)=\bar{e}_{\nu_{*}}(x, y) .
$$

Proof. Because $s_{\nu_{*}}(x, y)=\bar{s}_{\nu_{*}}(x, y)$, their negated forms are equivalent [17]. That is,

$$
e_{\nu_{*}}(x, y)=\eta_{\nu_{*}}\left(s_{\nu_{*}}(x, y)\right)=\eta_{\nu_{*}}\left(\bar{s}_{\nu_{*}}(x, y)\right)=\bar{e}_{\nu_{*}}(x, y)
$$


Proposition 4. The following identity is valid:

$$
e_{\nu_{*}}^{(1)}(x, y)=e_{\nu_{*}}^{(2)}(x, y)
$$

Proof. Because $s_{\nu_{*}, 1}(x, y)=s_{\nu_{*}, 2}(x, y)$ (see [17]), their negated forms are the same.

In a continuous-valued logic, (23) and (24) are not necessarily equal. Let $c(x, y)=x y, d(x, y)=x+y-x y$ and $\eta(x)=1-x$

$((1-x)+y-(1-x) y)(1-y+x-(1-y) x) \neq x y+(1-x)(1-y)-x y(1-y)(1-y)$

In the article by Bustince et al. [9], proposition 33 states that $e_{1}(x, y)$ is an equivalence operator. Here, we show that $e_{1}(x, y)$ is the same operator as that in the classical definition.

Definition 6. The Pliant equivalence operator is

$$
e_{\nu_{*}}(x, y)=f^{-1}\left(f^{2}\left(\nu_{*}\right) \frac{f(x)+f(y)}{f^{2}\left(\nu_{*}\right)+f(x) f(y)}\right),
$$

when $f\left(\nu_{*}\right)=1$, then $e_{\nu_{*}}(x, y)=e(x, y)$ and

$$
e(x, y)=f^{-1}\left(\frac{f(x)+f(y)}{1+f(x) f(y)}\right) .
$$

Definition 7. We will say $x$ and $y$ are threshold equivalent iff

$$
e_{\nu_{*}}(x, y)>\nu_{*} .
$$

When two facts are probably true, their equivalence is probably true and the same holds for the converse.

Remark 1. From this result we can infer that two values are in the same $\nu_{*}$ equivalence class if either $x>\nu_{*}$ and $y \geq \nu_{*}$ or $x<\nu_{*}$ and $y<\nu_{*}$.

The Pliant equivalence operator (30) satisfies the threshold property.

Proposition 5. The following inequalities are true:

$$
e_{\nu_{*}}(x, x)>\nu_{*} \quad e_{\nu_{*}}\left(\eta_{\nu_{*}}(x), x\right)<\nu_{*} \quad x \neq \nu_{*}
$$


Proof. Using the definition of $e_{\nu_{*}}(x, y)$, we have to prove that

$$
f^{-1}\left(\frac{2 f(x)}{f^{2}\left(\nu_{*}\right)+f^{2}(x)}\right)>\nu_{*}
$$

and

$$
f^{-1}\left(f^{2}\left(\nu_{*}\right) \frac{f(x)+\frac{f^{2}\left(\nu_{*}\right)}{f(x)}}{f^{2}\left(\nu_{*}\right)+\frac{f^{2}\left(\nu_{*}\right)}{f(x)} f(x)}\right)=\left(\frac{1}{2}\left(f(x)+\frac{f^{2}\left(\nu_{*}\right)}{f(x)}\right)\right)<\nu_{*} .
$$

From the fact that $f$ is a strictly decreasing function, we get, from (32) and (33), that

$$
2 f\left(\nu_{*}\right) f(x)<f^{2}\left(\nu_{*}\right)+f(x)
$$

Proposition 6. If $x>\nu_{*}$ and $y>\nu_{*}$, then $e_{\nu_{*}}(x, y)>\nu_{*}$;

$$
\text { and if } x<\nu_{*} \text { and } y<\nu_{*} \text {, then } e_{\nu_{*}}(x, y)<\nu_{*} \text {. }
$$

Proof. If $x>\nu_{*}$ and $y>\nu_{*}$, then $f(x)<f\left(\nu_{*}\right)$ and $f(y)<f\left(\nu_{*}\right)$. Because $f\left(\nu_{*}\right)-f(x)>0$

$$
f\left(\nu_{*}\right)\left(f\left(\nu_{*}\right)-f(x)\right)>f(y)\left(f\left(\nu_{*}\right)-f(x)\right)
$$

From this we see that

$$
f\left(\nu_{*}\right)(f(x)+f(y))<f^{2}\left(\nu_{*}\right)+f(x) f(y)
$$

Hence,

$$
e_{\nu_{*}}(x, y)=f^{-1}\left(f^{2}\left(\nu_{*}\right) \frac{f(x)+f(y)}{f^{2}\left(\nu_{*}\right)+f(x) f(y)}\right)>\nu_{*}
$$

Proposition 7. The following threshold property is valid for the Pliant equivalence operator

$e_{\nu_{*}}(x, y)>\nu_{*} \quad$ iff $\quad\left(x<\nu_{*} \quad\right.$ and $\left.\quad y<\nu_{*}\right) \quad$ or $\quad\left(x>\nu_{*} \quad\right.$ and $\left.\quad y>\nu_{*}\right)$

Proof. By definition,

$$
e_{\nu_{*}}(x, y)=f^{-1}\left(f^{2}\left(\nu_{*}\right) \frac{f(x)+f(y)}{f^{2}\left(\nu_{*}\right)+f(x) f(y)}\right)>\nu_{*}
$$

From this, we get

$$
f\left(\nu_{*}\right)(f(x) f(y))<f^{2}\left(\nu_{*}\right)+f(x) f(y) .
$$

Hence, 


$$
\begin{gathered}
\frac{f(y)}{f\left(\nu_{*}\right)}-1<\frac{f(x)}{f\left(\nu_{*}\right)}\left(\frac{f(y)}{f\left(\nu_{0}\right)}-1\right) . \\
\text { If } \frac{f(y)}{f\left(\nu_{*}\right)}-1>0 \text {, then } \frac{f(x)}{f\left(\nu_{*}\right)}>1 \text {, and if } \frac{f(y)}{f\left(\nu_{*}\right)}-1<1 \text {, then } \frac{f(x)}{f\left(\nu_{*}\right)}<1 .
\end{gathered}
$$

Proposition 8. The Pliant equivalence operator has the following properties:

1. $e_{\nu_{*}}(x, y)=e_{\nu_{*}}(y, x)$ commutativity

2. $e_{\nu_{*}}(1,1)=1, \quad e_{\nu_{*}}(0,0)=1$

$$
e_{\nu_{*}}(0,1)=0, \quad e_{\nu_{*}}(1,0)=0
$$

compatibility, $i$. e. compatible with the two-valued logic case

3. $e_{\nu_{*}}(1, x)=x \quad e_{\nu_{*}}(x, 1)=x \quad$ boundary conditions are fulfilled

$$
e_{\nu_{*}}(0, x)=\eta_{\nu_{*}}(x) \quad e_{\nu_{*}}(x, 0)=\eta_{\nu_{*}}(x)
$$



4. a) $e_{\nu_{*}}\left(\nu_{*}, \nu_{*}\right)=\nu_{*}$
fix point property
4. b) $e_{\nu_{*}}\left(\nu_{*}, x\right)=\nu_{*}$
$e_{\nu_{*}}\left(x, \nu_{*}\right)=\nu_{*}$
5. a) $\quad \arg \min _{x} e_{\nu_{*}}(x, x)=\nu_{*}$
minimum property of the equiva- lence operator
5. b) $\quad \arg \max _{x} e_{\nu_{*}}\left(x, \eta_{\nu_{*}}(x)\right)=\nu_{*}$
maximum property of the equiva- lence operator
6. $\quad e_{\nu_{*}}\left(\eta_{\nu_{*}}(x), \eta_{\nu_{*}}(y)\right)=e_{\nu_{*}}(x, y)$
invariance property with nega- tion
7. $\quad e_{\nu_{*}}\left(x, \eta_{\nu_{*}}(x)\right)=\bar{c}\left(x, \eta_{\nu_{*}}(x)\right)$
connection between equivalence and the conjunctive operators when $x=y$
8. $\quad \eta_{\nu_{*}}\left(e_{\nu_{*}}(x, y)\right)=e_{\nu_{*}}\left(\eta_{\nu_{*}}(x), y\right)=s_{\nu_{*}}(x, y)$
relationship between equivalence operator and symmetric differ- ence operator
$\eta_{\nu_{*}}\left(e_{\nu_{*}}(x, y)\right)=e_{\nu_{*}}\left(x, \eta_{\nu_{*}}(y)\right)=s_{\nu_{*}}(x, y)$
9. $\quad e_{\nu_{*}}\left(x, e_{\nu_{*}}(y, z)\right)=e_{\nu_{*}}\left(e_{\nu_{*}}(x, y), z\right)$
associativity
10. $\sup (c(x, y)) \leq \inf \left(e_{\nu_{*}}(x, y)\right)$
B. Moser [30]
11. $\quad c(x, y) \leq e_{\nu_{*}}(x, y)$
M. Cirić [11] property

Proof.

1. The proof of 1 is obvious.

2. $e_{\nu_{*}}(1,1)=f^{-1}\left(f^{2}\left(\nu_{*}\right) \frac{2 f(1)}{f^{2}\left(\nu_{*}\right)+f^{2}(1)}\right)=1$, because $f(1)=0, \quad f^{-1}(0)=1$

$$
\begin{aligned}
& e_{\nu_{*}}(0,0)=f^{-1}\left(f^{2}\left(\nu_{*}\right) \frac{2}{\frac{f^{2}\left(\nu_{*}\right)}{f(0)}+f(0)}\right)=1, \text { because } f(0)=\infty, \quad f^{-1}(0)=1 \\
& e_{\nu_{*}}(0,1)=e_{\nu_{*}}(1,0)=f^{-1}\left(f^{2}\left(\nu_{*}\right) \frac{f(0)+f(1)}{f^{2}\left(\nu_{*}\right)+f(0) f(1)}\right)=0, \text { because } f(0)=\infty
\end{aligned}
$$

3. $e_{\nu_{*}}(1, x)=f^{-1}\left(f^{2}\left(\nu_{*}\right) \frac{f(x)+f(1)}{f^{2}\left(\nu_{*}\right)+f(x) f(1)}\right)=x$ 


$$
\begin{gathered}
e_{\nu_{*}}(0, x)=e_{\nu_{*}}\left(\eta_{\nu_{*}}(1), x\right)=e_{\nu_{*}}\left(1, \eta_{\nu_{*}}(x)\right)=\eta_{\nu_{*}}(x) \\
e_{\nu_{*}}\left(0, \eta_{\nu_{*}}(x)\right)=f^{-1}\left(f^{2}\left(\nu_{*}\right) \frac{f(0)+\frac{f^{2}\left(\nu_{*}\right)}{f(x)}}{f^{2}\left(\nu_{*}\right)+\frac{f^{2}\left(\nu_{*}\right)}{f(x)} f(0)}\right)= \\
=f^{-1}\left(f^{2}\left(\nu_{*}\right) \frac{f(x)+\frac{f^{2}\left(\nu_{*}\right)}{f(0)}}{f^{2}\left(\nu_{*}\right)+\frac{f^{2}\left(\nu_{*}\right) f(x)}{f(0)}}\right)=x
\end{gathered}
$$

4. a) $e_{\nu_{*}}\left(\nu_{*}, \nu_{*}\right)=\nu_{*}$

$$
f^{-1}\left(f^{2}\left(\nu_{*}\right) \frac{2 f\left(\nu_{*}\right)}{2 f^{2}\left(\nu_{*}\right)}\right)=\nu_{*}
$$

4. b) $f^{-1}\left(f^{2}\left(\nu_{*}\right) \frac{f(x)+f(y)}{f^{2}\left(\nu_{*}\right)+f(x) f(y)}\right)=\nu_{*}$ if and only if $f(y)\left(f(x)-f\left(\nu_{*}\right)\right)=f\left(\nu_{*}\right)\left(f(x)-f\left(\nu_{*}\right)\right)$

5. a) $\arg \min \left(e_{\nu_{*}}(x, x)\right)=\arg \max \left(\frac{f(x)}{f^{2}\left(\nu_{*}\right)+f^{2}(x)}\right)=$

$$
\arg \min \left(\frac{f^{2}(x)}{f(x)}+f(x)\right)=\nu_{*}
$$

5. b) $\arg \max \left(e_{\nu_{*}}\left(x, \eta_{\nu_{*}}(x)\right)\right)=\arg \min \left(\frac{f(x)+\frac{f^{2}\left(\nu_{*}\right)}{f(x)}}{f^{2}\left(\nu_{*}\right)+\frac{f^{2}\left(\nu_{*}\right)}{f^{2}(x)} f(x)}\right)=\nu_{*}$

6. $f^{-1}\left(f\left(\nu_{*}\right) \frac{\frac{f^{2}\left(\nu_{*}\right)}{f(x)}+\frac{f^{2}\left(\nu_{*}\right)}{f(y)}}{f^{2}\left(\nu_{*}\right)+\frac{f^{2}\left(\nu_{*}\right) f\left(\nu_{*}\right)}{f(x), f(y)}}\right)=f^{-1}\left(f\left(\nu_{*}\right) \frac{f(x)+f(y)}{f^{2}\left(\nu_{*}\right)+f(x) f(y)}\right)$

7. $f^{-1}\left(f^{2}\left(\nu_{*}\right) \frac{f(x)+\frac{f^{2}\left(\nu_{*}\right)}{f(x)}}{f^{2}\left(\nu_{*}\right)+f(x) \frac{f^{2}\left(\nu_{*}\right)}{f(x)}}\right)=f^{-1}\left(\frac{1}{2}\left(f(x)+\frac{f^{2}\left(\nu_{*}\right)}{f(x)}\right)\right)$

8. $f^{-1}\left(\frac{f^{2}\left(\nu_{*}\right)}{f^{2}\left(\nu_{*}\right)+\frac{f(x)+f(y)}{f^{2}\left(\nu_{*}\right)+f(x) f(y)}}\right)=f^{-1}\left(f^{2}\left(\nu_{*}\right) \frac{\frac{f^{2}\left(\nu_{*}\right)}{f(x)}+f(y)}{f^{2}\left(\nu_{*}\right)+\frac{f^{2}\left(\nu_{*}\right)}{f(x)} f(y)}\right)$

9. $e_{\nu_{*}}(x, c(y, z))=$

$$
\begin{aligned}
& =f^{-1}\left(f^{2}\left(\nu_{*}\right) \frac{f(x)+f^{2}\left(\nu_{*}\right) \frac{f(y)+f(z)}{f^{2}\left(\nu_{*}\right)}+f(y) f(z)}{f^{2}\left(\nu_{*}\right)+f(x) f^{2}\left(\nu_{*}\right) \frac{f(y)+f(z)}{f^{2}\left(\nu_{*}\right)+f(y) f(z)}}\right)= \\
& =f^{-1}\left(\frac{f^{2}\left(\nu_{*}\right)(f(x)+f(y)+f(z))+f(x) f(y) f(z)}{f^{2}\left(\nu_{*}\right) f^{2}\left(\nu_{*}\right) f(x) f(y)+f(x) f(z)+f(y) f(z)}\right)= \\
& =f^{-1}\left(\frac{f^{2}\left(\nu_{*}\right) \frac{f(x)+f(y)}{f^{2}\left(\nu_{*}\right)+f(x) f(y)}+f(z)}{f^{2}\left(\nu_{*}\right)+f(z) f^{2}\left(\nu_{*}\right) \frac{f(x)+f(y)}{f^{2}\left(\nu_{*}\right)+f(x) f(y)}}\right)=e_{\nu_{*}}\left(e_{\nu_{*}}(x, y), z\right)
\end{aligned}
$$

10. In Moser's article [30], this property has a crucial rule and it is the socalled $\boldsymbol{F P}$ property.

$$
\sup \left(f^{-1}(f(x)+f(y))\right) \leq \inf \left(f^{-1}\left(f^{2}\left(\nu_{*}\right) \frac{f(x)+f(y)}{f^{2}\left(\nu_{*}\right)+f(x) f(y)}\right)\right), \quad \text { so }
$$




$$
\begin{array}{r}
\inf (f(x)+f(y)) \geq \sup \left(f\left(\nu_{*}\right) \frac{\frac{f(x)}{f\left(\nu_{*}\right)}+\frac{f(y)}{f\left(\nu_{*}\right)}}{1 \frac{f(x)}{f\left(\nu_{*}\right)} \frac{f(y)}{f\left(\nu_{*}\right)}}\right) \\
\text { Let } X=\frac{f(x)}{f\left(\nu_{*}\right)} \text { and } Y=\frac{f(y)}{f\left(\nu_{*}\right)} . \quad \text { Then we have } \\
\inf (X+Y) \geq \sup \left(\frac{X+Y}{1+X Y}\right) \\
1 \geq \sup \left(\frac{1}{1+X Y}\right) \\
\text { 11. } f^{-1}(f(x)+f(y)) \leq f^{-1}\left(f^{2}\left(\nu_{*}\right) \frac{f(x)+f(y)}{f^{2}\left(\nu_{*}\right)+f(x) f(y)}\right) \\
f^{2}\left(\nu_{*}\right)+f(x) f(y) \geq f^{2}\left(\nu_{*}\right)
\end{array}
$$

Because $e(x, y)$ is associative, there is an additive generator function of the operator based on Aczél's theorem:

$$
e_{\nu_{*}}(x, y)=E^{-1}(E(x)+E(y))
$$

Proposition 9. We show that $E(x)$ has the form

$$
E(x)=\ln \left(\frac{f\left(\nu_{*}\right)+f(x)}{f\left(\nu_{*}\right)-f(x)}\right)
$$

Proof. From (36), we get

$$
E^{-1}(x)=f^{-1}\left(f\left(\nu_{*}\right) \frac{e^{x}-1}{e^{x}+1}\right) .
$$

Using (36), we have

$$
\begin{aligned}
e_{\nu_{*}}(x, y) & =f^{-1}\left(f\left(\nu_{*}\right) \frac{\frac{f\left(\nu_{*}\right)+f(x)}{f\left(\nu_{*}\right)-f(x)} \frac{f\left(\nu_{*}\right)+f(y)}{f\left(\nu_{*}\right)-f(y)}-1}{\frac{f\left(\nu_{*}\right)+f(x)}{f\left(\nu_{*}\right)-f(x)} \frac{f\left(\nu_{*}\right)+f(y)}{f\left(\nu_{*}\right)-f(y)}+1}\right)= \\
& =f^{-1}\left(f^{2}\left(\nu_{*}\right) \frac{f(x)+f(y)}{f^{2}\left(\nu_{*}\right)+f(x) f(y)}\right) .
\end{aligned}
$$


Remark 2. Because $e_{\nu_{*}}(x, y)$ is associative, we can extend it to $n$ variables. By definition,

$$
e_{\nu_{*}}(\boldsymbol{x})=E^{-1}\left(\sum_{i=1}^{n} E\left(x_{i}\right)\right)=f^{-1}\left(f\left(\nu_{*}\right) \frac{\prod_{i=1}^{n} \frac{f\left(\nu_{*}\right)+f\left(x_{i}\right)}{f\left(\nu_{*}\right)-f\left(x_{i}\right)}-1}{\prod_{i=1}^{n} \frac{f\left(\nu_{*}\right)+f\left(x_{i}\right)}{f\left(\nu_{*}\right)-f\left(x_{i}\right)}+1}\right)
$$

Then we get

$$
e(\boldsymbol{x})=f^{-1}\left(f\left(\nu_{*}\right) \frac{\prod_{i=1}^{n}\left(f\left(\nu_{*}\right)+f\left(x_{i}\right)\right)-\prod_{i=1}^{n}\left(f\left(\nu_{*}\right)-f\left(x_{i}\right)\right)}{\prod_{i=1}^{n}\left(f\left(\nu_{*}\right)+f\left(x_{i}\right)\right)+\prod_{i=1}^{n}\left(f\left(\nu_{*}\right)-f\left(x_{i}\right)\right)}\right)
$$

Remark 3. The weighted form of the equivalence operator is, by definition,

$$
e(\boldsymbol{w}, \boldsymbol{x})=E^{-1}\left(\sum_{i=1}^{n} w_{i} E\left(x_{i}\right)\right)=f^{-1}\left(f\left(\nu_{*}\right) \frac{\prod_{i=1}^{n}\left(\frac{f\left(\nu_{*}\right)+f\left(x_{i}\right)}{f\left(\nu_{*}\right)-f\left(x_{i}\right)}\right)^{w_{i}}-1}{\prod_{i=1}^{n}\left(\frac{f\left(\nu_{*}\right)+f\left(x_{i}\right)}{f\left(\nu_{*}\right)-f\left(x_{i}\right)}\right)^{w_{i}}+1}\right)
$$

Hence,

$$
e(\boldsymbol{w}, \boldsymbol{x})=f^{-1}\left(f\left(\nu_{*}\right) \frac{\prod_{i=1}^{n}\left(f\left(\nu_{*}\right)+f\left(x_{i}\right)\right)^{w_{i}}-\prod_{i=1}^{n}\left(f\left(\nu_{*}\right)-f\left(x_{i}\right)\right)^{w_{i}}}{\prod_{i=1}^{n}\left(f\left(\nu_{*}\right)+f\left(x_{i}\right)\right)^{w_{i}}+\prod_{i=1}^{n}\left(f\left(\nu_{*}\right)-f\left(x_{i}\right)\right)^{w_{i}}}\right) .
$$

\section{Transitivity of the equivalence operator}

The composition of two relations is defined in the following way:

$$
(R \circ S)(x, y)=\bigvee_{a} c(R(x, a), S(a, y))
$$

The composition of one relation is when $c(x, y)$ is a strict monotonously increasing t-norm:

$$
(R \circ R)(x, y)=\bigvee_{a} f^{-1}(f(R(x, a))+f(R(a, y)))
$$

Definition 8. The $R$ relation is t-transitive (composition) if and only if

$$
R \circ R \leq R
$$


Proposition 10. The Pliant equivalence $e_{\nu_{*}}(x, y)$ is t-transitive (in our case c-transitive).

Proof. By definition,

$\left(e_{\nu_{*}} \circ e_{\nu_{*}}\right)(x, y)=f^{-1}\left(\bigwedge_{a} f\left(e_{\nu_{*}}(x, a)\right)+f\left(e_{\nu_{*}}(a, y)\right)\right) \leq f^{-1}\left(f^{2}\left(\nu_{*}\right) \frac{f(x)+f(y)}{f^{2}\left(\nu_{*}\right)+f(x) f(y)}\right)=e_{\nu_{*}}(x, y)$

Because $f$ is strictly decreasing, we need to show that

$$
\bigvee_{a} \frac{f(x)+f(a)}{f^{2}\left(\nu_{*}\right)+f(x) f(y)}+\frac{f(y)+f(a)}{f^{2}\left(\nu_{*}\right)+f(y) f(a)} \geq \frac{f(x)+f(y)}{f^{2}\left(\nu_{*}\right)+f(x) f(y)}
$$

Let $X=\frac{f(x)}{f\left(\nu_{*}\right)}, \quad Y=\frac{f(y)}{f\left(\nu_{*}\right)}, \quad A=\frac{f(a)}{f\left(\nu_{*}\right)}, \quad X, Y, A \in[0, \infty]$

$$
\bigwedge_{A}\left(\frac{X+A}{1+X A}+\frac{Y+A}{1+Y A}\right) \geq \frac{X+Y}{1+X Y}
$$

Let us introduce the function

$$
F_{X}(A)=\frac{X+A}{1+X A}
$$

$F_{X}(A)$ is strictly increasing when $X \leq 1$ and strictly decreasing when $X>1$.

As $F_{X}(0)=X$ and $F_{X}(\infty)=\frac{1}{X}$, we have that

$$
\min _{A}\left(F_{X}(A)\right)=\min \left(X, \frac{1}{X}\right)
$$

Next, we have to prove that
a) $X+Y \geq \frac{X+Y}{1+X Y}$
b) $X+\frac{1}{Y} \geq \frac{X+Y}{1+X Y}$
c) $\frac{1}{X}+\frac{1}{Y} \geq \frac{X+Y}{1+X Y}$
a), b) and c) are trivial.

Definition 9. $e_{\nu_{*}}(x, y)$ is threshold transitive if $e_{\nu_{*}}(x, y) \geq \nu_{*}$ and $e_{\nu_{*}}(y, z) \geq$ $\nu_{*}$. Then $e_{\nu_{*}}(x, z) \geq \nu_{*}$.

Proposition 11. $e_{\nu_{*}}(x, y)$ is threshold transitive. 
Proof. From the definition,

$$
e_{\nu_{*}}(x, y)=f^{-1}\left(f^{2}\left(\nu_{*}\right) \frac{f(x)+f(y)}{f(x) f(y)}\right)
$$

We can write $e_{\nu_{*}}(x, y)$ in the following way:

$$
e_{\nu_{*}}(x, y)=f^{-1}\left(f\left(\nu_{*}\right) \frac{\frac{f(x)}{f\left(\nu_{*}\right)}+\frac{f(y)}{f\left(\nu_{*}\right)}}{1+\frac{f(x) f(y)}{f^{2}\left(\nu_{*}\right)}}\right) \geq \nu_{*}
$$

Since $f(x)$ is a strictly decreasing function, using the notation $X=\frac{f(x)}{f\left(\nu_{*}\right)}$, $Y=\frac{f(y)}{f\left(\nu_{*}\right)}$ and $Z=\frac{f(z)}{f\left(\nu_{*}\right)}$, we need to prove that if

$$
\begin{aligned}
& X+Y \leq 1+X Y \\
& Y+Z \leq 1+Y Z
\end{aligned}
$$

then

$$
X+Z \leq 1+X Z
$$

where $X, Y, Z \geq 0$.

The inequalities (39) can be rewritten as:

$$
Y-1 \leq X(Y-1)
$$

This is valid iff

$$
1 \leq Y \quad \text { and } \quad 1 \leq X
$$

or

$$
0 \leq X \leq 1 \quad \text { and } \quad 0 \leq Y \leq 1
$$

From (39), if $1 \leq X$, then $1 \leq Y$ from (40), if $1 \leq Y$, then $1 \leq Z$ so (41) is valid. In this way, we can prove the case when $X \leq 1$.

\section{Equivalence and symmetric difference operators in the Dombi op-} erator case

Let us use the generator function in the Dombi operator case:

$$
f(x)=\left(\frac{1-x}{x}\right)^{\alpha} \quad \text { and } \quad f^{-1}(x)=\frac{1}{1+x^{\frac{1}{\alpha}}}
$$


and let

$$
\begin{aligned}
& K_{1}(\mathbf{w}, \mathbf{x})=\prod_{i=1}^{n}\left(\left(1-\nu_{*}\right)^{\alpha} x_{i}^{\alpha}+\nu_{*}^{\alpha}\left(1-x_{i}\right)^{\alpha}\right)^{w_{i}} \\
& K_{2}(\mathbf{w}, \mathbf{x})=\prod_{i=1}^{n}\left(\left(1-\nu_{*}\right)^{\alpha} x_{i}^{\alpha}-\nu_{*}^{\alpha}\left(1-x_{i}\right)^{\alpha}\right)^{w_{i}}
\end{aligned}
$$

then

$$
e_{\nu_{*}}^{(\alpha)}(\mathbf{w}, \mathbf{x})=\frac{1}{1+\frac{1-\nu_{*}}{\nu_{*}}\left(\frac{K_{1}-K_{2}}{K_{1}+K_{2}}\right)^{\frac{1}{\alpha}}}=\frac{\nu_{*}\left(K_{1}+K_{2}\right)^{\frac{1}{\alpha}}}{\nu_{*}\left(K_{1}+K_{2}\right)^{\frac{1}{\alpha}}+\left(1-\nu_{*}\right)\left(K_{1}-K_{2}\right)^{\frac{1}{\alpha}}}
$$

When $\alpha=1$ and $\nu_{*}=\frac{1}{2}$

$$
\begin{gathered}
K_{1}(\mathbf{w}, \mathbf{x})=1 \quad \text { and } \quad K_{2}(\mathbf{w}, \mathbf{x})=\prod\left(1-2 x_{i}\right)^{w_{i}} \\
e(\mathbf{w}, \mathbf{x})=\frac{1}{2}\left(1+\prod\left(1-2 x_{i}\right)^{w_{i}}\right)
\end{gathered}
$$

When $n=2$,

$$
\begin{gathered}
K_{1}=\left(\left(1-\nu_{*}\right)^{\alpha} x^{\alpha}+\nu_{*}^{\alpha}(1-x)^{\alpha}\right)^{w_{1}}\left(\left(1-\nu_{*}\right)^{\alpha} y^{\alpha}+\nu_{*}^{\alpha}(1-y)^{\alpha}\right)^{w_{2}} \\
K_{2}=\left(\left(1-\nu_{*}\right)^{\alpha} x^{\alpha}-\nu_{*}^{\alpha}(1-x)^{\alpha}\right)^{w_{1}}\left(\left(1-\nu_{*}\right)^{\alpha} y^{\alpha}-\nu_{*}^{\alpha}(1-y)^{\alpha}\right)^{w_{2}} \\
e_{\nu_{*}}^{(\alpha)}\left(w_{1}, x ; w_{2}, y\right)=\frac{\nu_{*}\left(K_{1}+K_{2}\right)^{\frac{1}{\alpha}}}{\nu_{*}\left(K_{1}+K_{2}\right)^{\frac{1}{\alpha}}+\left(1-\nu_{*}\right)\left(K_{1}-K_{2}\right)^{\frac{1}{\alpha}}}
\end{gathered}
$$

When $n=2$ and $\nu_{*}=\frac{1}{2}$,

$$
\begin{gathered}
K_{1}=\left(x^{\alpha}+(1-x)^{\alpha}\right)^{w_{1}}\left(y^{\alpha}+(1-y)^{\alpha}\right)^{w_{2}} \\
K_{2}=\left(x^{\alpha}-(1-x)^{\alpha}\right)^{w_{1}}\left(y^{\alpha}-(1-y)^{\alpha}\right)^{w_{2}} \\
e^{(\alpha)}\left(w_{1}, x ; w_{2}, y\right)=\frac{\left(K_{1}+K_{2}\right)^{\frac{1}{\alpha}}}{\left(K_{1}+K_{2}\right)^{\frac{1}{\alpha}}+\left(K_{1}-K_{2}\right)^{\frac{1}{\alpha}}}
\end{gathered}
$$

When $n=2, \nu_{*}=\frac{1}{2}$ and $\alpha=1 \quad$ (see Figure 2)

$$
\begin{gathered}
K_{1}=1, \quad K_{2}=(2 x-1)^{w_{1}}(2 y-1)^{w_{2}} \\
e\left(w_{1}, x ; w_{2}, y\right)=\frac{1}{2}\left(1+(2 x-1)^{w_{1}}(2 y-1)^{w_{2}}\right)
\end{gathered}
$$


When $n=2, \nu_{*}=\frac{1}{2} \alpha=1, w_{1}=1 \quad$ and $w_{2}=1(1)$

$$
e(x, y)=\frac{1}{2}(1+(2 x-1)(2 y-1))=x y+(1-x)(1-y)
$$

Definition 10. The weighted equivalence of $\boldsymbol{x}$ and $\boldsymbol{y}$ is

$$
e_{\nu_{*}}(\boldsymbol{x}, \boldsymbol{y})=\sum_{i=1}^{n} w_{i} e_{\nu_{*}}\left(x_{i}, y_{i}\right)
$$

where $w_{i} \geq 0$ and $\sum_{i=1}^{n} w_{i}=1$.

And a special case:

$$
e_{D}(\boldsymbol{x}, \boldsymbol{y})=\sum_{i=1}^{n} w_{i}\left(1+\left(2 x_{i}-1\right)^{u}\left(2 y_{i}-1\right)^{v}\right)
$$

This is none other than the strong equality index when $w_{i}=\frac{1}{n} \quad \forall i$.

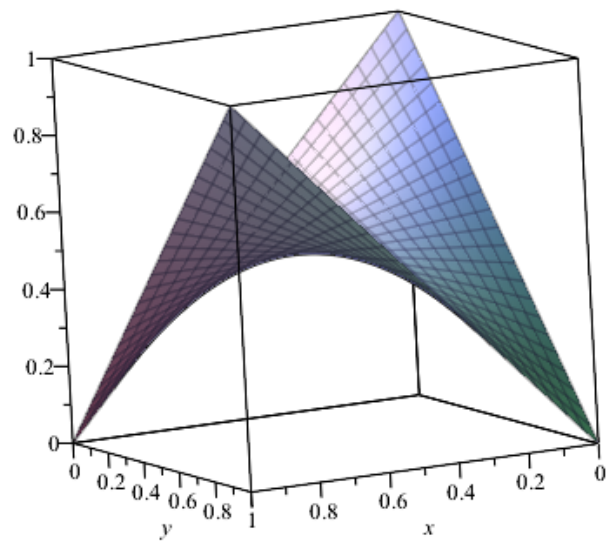

Figure 1: Asymmetric equivalence operator when $n=2, \alpha=1, \nu_{*}=1 / 2 u=1 / 2$ and $v=1 / 2$

The asymmetric equivalence operator equation is (as in Equation 27)

$$
e_{\nu_{*}}^{(\alpha)}(u, x ; v, y)=\frac{1}{1+\left(2 \nu_{*}^{2 \alpha} \frac{v(1-x)^{\alpha} y^{\alpha}+u(1-y)^{\alpha} x^{\alpha}}{\left(1-\nu_{*}\right)^{2 \alpha} x^{\alpha} y^{\alpha}+\nu_{*}^{2 \alpha}(1-x)^{\alpha}(1-y)^{\alpha}}\right)}
$$

When $\alpha=1$, then

$$
e_{\nu_{*}}(u, x ; v, y)=\frac{1}{1+\left(2 \nu_{*}^{2} \frac{v(1-x)+u(1-y)}{\left(1-\nu_{*}\right)^{2} x y+\nu_{*}^{2}(1-x)(1-y)}\right)}
$$




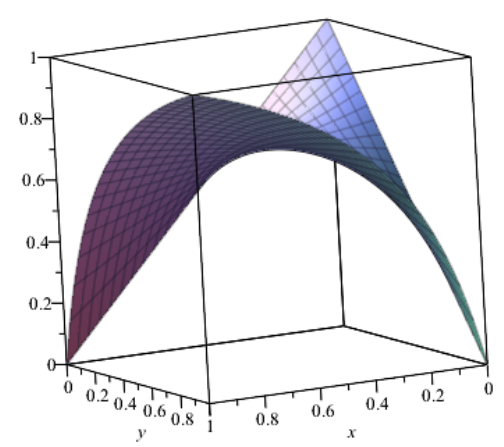

Figure 2: Asymmetric equivalence operator when $\nu_{*}=1 / 3, \alpha=1, u=1 / 2$ and $v=1 / 2$

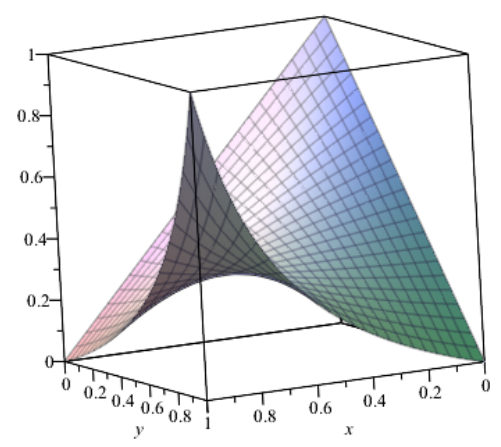

Figure 3: Asymmetric equivalence operator when $\quad \nu_{*}=2 / 3, \alpha=1, u=1 / 2$ and $v=1 / 2$

see Figures 2, 3.

When $\nu_{*}=\frac{1}{2}$ and $\alpha=1$,

$$
e(u, x ; v, y)=\frac{1}{1+\left(\frac{v(1-x)+u(1-y)}{x y+(1-x)(1-y)}\right)}
$$

\section{Summary}

It was an interesting open problem to determine (independently of the definition of equivalence) which t-norms and t-conorms and strong negation operators of two variables are associative operations on the unit interval. In this article, we showed that the Pliant operators have just this property.

We demonstrated that the two different operators $e_{1}(x, y), e_{2}(x, y)$ are equivalent. After, we proved that the desired properties of the equivalence exist. We showed that the Pliant equivalence is associative and it has other nice properties. Then we gave a parametrical form of these operators to illustrate their flexibility in problem solving. We gave a parametrical form of the equivalence operator. When $\alpha=1$, the equivalence operator is a very simple expression and it has many possible applications, such as in image processing and signal processing. With this, we can also measure the degree of overlap.

\section{Acknowledgement}

The author is grateful to all anonymous referees whose comments and suggestions have significantly improved our original version of this paper. This 
study was partially supported by "Telemedicine-focused research activities on the field of Mathematics, Informatics and Medical sciences", TÁMOP-4.2.2.A11/1/KONV-2012-0073 
[1] A. Alsina "On the symmetric difference of fuzzy sets" Fuzzy Sets and Systems, vol. 153, issue 2, pp. 181-194, 2005

[2] B. De Baets, R. Mesiar, "T-partitions", Fuzzy Sets and Systems, vol. 97, pp. 211-223, 1998

[3] B. De Baets, R. Mesiar, "Metrics and T-equalities" Journal of Mathematical Analysis and its Applications, vol. 267, pp. 531-547, 2002

[4] B. De Baets, R. Mesiar, "Pseudo-metrics and T-equivalences", Journal Fuzzy Mathemathics, vol. 5, issue 2, pp. 471-481, 1997

[5] I. Bloch, "On fuzzy distances and their use in image processing under imprecision". Pattern Recognition, vol. 32, issue 11, 1999

[6] U. Bodenhofer "Representations and constructions of similarity-based fuzzy orderings" Fuzzy Sets Systems, vol. 137, issue 1, pp. 113-136, 2003

[7] D. Boixader, J. Jacas, J. Recasens, "Transitive closure and betweenness relations" Fuzzy Sets and Systems, vol. 120, pp. 415-422, 2001

[8] G. Boole, "The Mathematical Analysis of Logic" Macmillan, Barclay, and Macmillan, Cambridge, 1847

[9] H. Bustince Sola, J. Fernández, J. Sanz, M. Baczyński, R. Mesiar "Construction of strong equality index from implication operators" Fuzzy Sets and Systems, vol. 211, pp. 15-33, 2013

[10] P. Cintula, E. P. Klement, R. Mesiar and M. Navara. "Fuzzy logics with an additional involutive negation" Fuzzy Sets and Systems, vol. 161, pp. 390-411, 2010

[11] M. Ćirić, J. Ignjatović, S. Bogdanović, "Fuzzy equivalence relations and their equivalence classes", Fuzzy Sets and Systems, vol. 158, issue 12, pp. 1295-1313, 2007

[12] M. Demirci, "On many-valued partitions and many valued equivalence relations", International Journal of Uncertainty, Fuzziness KnowledgeBased Systems, vol. 11, pp. 235-253, 2003

[13] M. Demirci, "Representations of the extensions of many-valued equivalence relations", International Journal of Uncertainty, Fuzziness Knowledge-Based Systems, vol. 11, pp. 319-341, 2003

[14] M. Demirci, "Indistinguishability operators in measurement theory, Part I: Conversions of indistinguishability operators with respect to scales", International Journal of General Systems, vol. 32, pp. 415430,2003 
[15] M. Demirci, "Indistinguishability operators in measurement theory, Part II: Construction of indistinguishability operators based on probability distributions", International Journal of General Systems, vol. 32, pp. $431-458,2003$

[16] J. Dombi, "On a certain class of aggregative operators" Information Sciences, vol. 245, pp. 313-328, 2013

[17] J. Dombi, "On symmetric difference operators" submitted to Fuzzy Sets and Systems, 2013

[18] J. Dombi. "DeMorgan systems with an infinitely many negations in the strict monotone operator case" Information Sciences, vol. 181, issue 8, pp. 1440-1453, 2011

[19] J. Fodor, M. Roubens, "Fuzzy Preference Modelling and Multicriteria Decision Support", Kluwer Academic Publishers, Dordrecht, 1994

[20] J. Fodor, R. Klempous, C. P. S. Araujo "Recent Advances in Intelligent Engineering Systems", Springer, Berlin Heidelberg, 2012

[21] L. Foulloy, E. Benoit, "Building a class of fuzzy equivalence relations", Fuzzy Sets and Systems, vol. 157, issue 11, pp. 1417-1437, 2006

[22] S. Gottwald, "Fuzzy Sets and Fuzzy Logic", Vieweg, Braunschweig, 1993

[23] P. Hájek, "Metamathematics of Fuzzy Logic", Trends in Logic, vol. 4, Kluwer Academic Publishers, Dordrecht, 1998

[24] J. Jacas, "Similarity relations. The calculation of minimal generating families", Fuzzy Sets and Systems, vol. 35, pp. 151-162, 1990

[25] J. Jacas, J. Recasens, "Fuzzy T-transitive relations: eigenvectors and generators", Fuzzy Sets and Systems, vol. 72, pp. 147-154, 1995

[26] J. Jacas, J. Recasens, "Maps and isometries between indistinguishability operators", Soft Computing, vol. 6, pp. 14-20, 2002

[27] A. Kaufman, M.M. Gupta, "Introduction to Fuzzy Arithmetic". Van Nostrand Reinhold, NewYork, 1985

[28] E. P. Klement, R. Mesiar and E. Pap "Triangular Norms" Kluwer, Dordrecht, 2000

[29] D. Mihet,, V. Radu, "T-equalities as fuzzy non-Archimedean metrics and $\Delta^{+}$-fuzzy equivalences of type $S^{\prime \prime}$, Fuzzy Sets and Systems, vol. 157, issue 20, pp. 2751-2761, 2006

[30] B. Moser. "On the compactness of admissible transformations of fuzzy partitions in terms of T -equivalence relations". Fuzzy Sets Systems, vol. 160 , issue 1 , pp. 95-106, 2009 
[31] B. Moser, "A new approach for representing control surfaces by fuzzy rule bases", Ph.D. Thesis, Johannes Kepler Universitat Linz, 1995

[32] M. Rifqi, M. J. Lesot, M. Detyniecki, "Fuzzy order-equivalence for similarity measures". 27th North American Fuzzy Information Processing Society Annual Conference, (NAFIPS 2008), New York, 2008

[33] L. Valverde, "On the structure of F-indistinguishability operators", Fuzzy Sets and Systems, vol. 17, issue 3, pp. 313-328, 1985

[34] C. Vertan, N. Boujemaa, "Using fuzzy histograms and distances for color image retrieval". Proceedings of CIR 2000, Brighton, UK, 2000

[35] A. Yoshikawa, T. Nishimura, "Relationship between subjective degree of similarity and some similarity indices of fuzzy sets". T. Yamukawa, G. Matsumoto (Eds.), Methodologies for the Conception, Design and Application of Intelligent Systems, World Scientific Singapore, pp. 818821,1966

[36] L. A. Zadeh, "Similarity relations and fuzzy orderings", Information Science, vol. 3, pp. 177-200, 1971

[37] H. J. Zimmermann, "Fuzzy Sets, Decision Making and Expert Systems". Kluwer Academic Publishers, Boston MA, 1987 